\title{
On Social Event Organization
}

\author{
by
}

\author{
Keqian Li
}

B. Science, Tsinghua University, 2013

\section{A THESIS SUBMITTED IN PARTIAL FULFILLMENT \\ OF THE REQUIREMENTS FOR THE DEGREE OF}

\author{
Master of Science \\ in \\ FACULTY OF GRADUATE AND POSTDOCTORAL STUDIES \\ (Computer Science) \\ The University of British Columbia \\ (Vancouver) \\ April 2016 \\ (c) Keqian Li, 2016
}




\section{Abstract}

Online platforms, such as Meetup and Plancast, have recently become popular for planning gatherings and event organization. However, there is a surprising lack of studies on how to effectively and efficiently organize social events for a large group of people through those platforms. This thesis provides the first systematic study on key computational problem involved in organization of social events. We understand the Social Event Organization problem as assigning a set of events for a group of users to attend, where the users are socially connected with each other and have innate levels of interest in those events. We then introduce a set of formal definition of a restricted version of the problem and show that it is NP-hard and is hard to approximate. We propose efficient heuristic algorithms that improve upon simple greedy algorithms by incorporating the notion of phantom events and by using look-ahead estimation. Using synthetic datasets and three real datasets including those from the platforms Meetup and Plancast, we experimentally demonstrate that our greedy heuristics are scalable and furthermore outperform the baseline algorithms significantly in terms of achieving superior social welfare. 


\section{Preface}

This dissertation is the result of collaborative research based on a publication [17] in the 2014 KDD Conference, a joint work with Prof. Lakshmanan, Dr. Wei Lu, Dr. Smriti Bhagat, Prof. Cong Yu. I developed the theory and conducted the experiments under their guidance, with the help from them for paper presentation and introducing the problem as constrained optimization problem. 


\section{Table of Contents}

Abstract .......................... ii

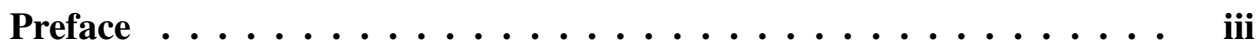

Table of Contents $\ldots \ldots \ldots \ldots \ldots \ldots$ iv

List of Tables $\ldots \ldots \ldots \ldots \ldots \ldots \ldots \ldots$ vi

List of Figures $\ldots \ldots \ldots \ldots \ldots \ldots \ldots$ vii

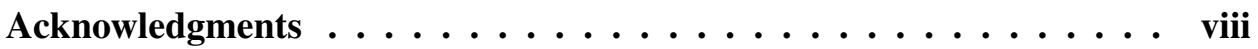

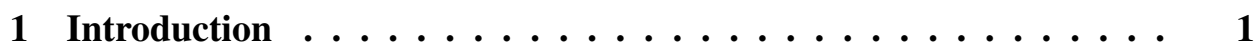

2 Related work $\ldots \ldots \ldots \ldots \ldots$

3 Social event organization $\ldots \ldots \ldots$

3.1 Problem definition $\ldots \ldots \ldots \ldots$

3.2 Hardness results . . . . . . . . . . . . . . . 13

4 Proposed solutions . . . . . . . . . . . . . . . . . 16

4.1 Baselines . . . . . . . . . . . . . . 17

4.2 Greedy with look ahead estimation . . . . . . . . . . 21

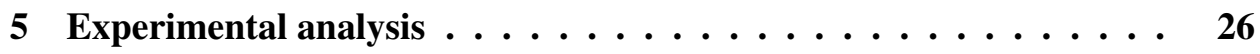

5.1 Experiments on synthetic datasets . . . . . . . . . . . 27

5.2 Experiments on real event datasets . . . . . . . . . 30 
6 Conclusions and future work $\ldots \ldots \ldots 33$

Bibliography ............................. 37 


\section{List of Tables}

Table $2.1 \quad$ Innate affinity for Example $3 \ldots \ldots \ldots$

Table 5.1 Running time (in seconds) of PADG and PCADG . . . . . . 29

Table 5.2 Running time (in seconds) of all algorithms with $10 \mathrm{~K}$ users and 100 events .................... 29 


\section{List of Figures}

Figure 5.1 Effects of parameter choices on the performance of PADG and PCADG . . . . . . . . . . . . . . . . 27

Figure 5.2 Distributions of social affinity values . . . . . . . . . . 28

Figure 5.3 Improvement of social welfare yielded by various algorithms over Random . . . . . . . . . . . . . . . . . . . . . 29

Figure 5.4 CDF showing regret ratio $\rho$ for different algorithms. . . . . 30 


\section{Acknowledgments}

Thank my advisor Professor Laks V.S. Lakshmanan who supervises my thesis research and my collaborator Mr. Wei Lu, Dr. Smriti Baghat, Dr. Cong Yu who gave tremendous helpful suggestions and helped with paper presentation and finally my family for their everlasting love and support. 


\section{Chapter 1}

\section{Introduction}

The problem of organizing social events for a large group of people can resonate with the academic community: we are all familiar with the "canned" social events offered by conference organizers, which are often a simple list of activities to choose from and attendance at each event is organically decided by people at the conference based on who they know and how interested they are in the events.

More broadly, event organization is one of the most important social activities online, with many companies, established giants and startups alike, such as Meetur ${ }^{1}$ and Plancast ${ }^{2}$, offering platforms for their users to plan and organize events. The social networks data and the data indicating the interests of the users on these platforms offer a rich setting in which events can be organized effectively and efficiently. Existing academic studies on this topic only focuses on specific area such as event detection through time series analysis (e.g., burst detection). The goal of this thesis is to provide a first systematic study on the problem of Social Event Organization, provide principled definitions and a platform of algorithmic solutions for addressing this problem.

The tasks of organizing various social events share many common characteristics. First, each individual user often has his or her unique preference for the events being offered, a numerical measure which we call a user's innate affinity towards an event. Innate affinities can be stated explicitly. For example, a user can mark his

\footnotetext{
${ }^{1}$ http://www.meetup.com

${ }^{2}$ http://www.plancast.com
} 
preference for a game of chess as 9 out of 10 . Or they can be categorically stated by the users and computed by the organizer. For example, a user can list outdoor activities in her profile and the organizer can deduce that she will likely prefer hiking to playing chess. Finally, they can be computed based on the past events that the user has participated in and enjoyed. Regardless of how innate affinities are computed, they are one of the two key ingredients for users' happiness, for purposes of successful organization of social events.

The other key ingredient is the social connections among users - individuals often enjoy an activity more if they attend it along with their friends or others whom they would like to be around with. This connection is often defined between a pair of users and we call this pairwise social affinity. Similar to innate affinities, social affinities can be stated explicitly by the users. For example, users on social networks explicitly provide their friendship connections. They can also be deduced based on users' interests or past activities. For instance, two users who share lots of interests and past activities are more likely to enjoy each other's company. Thus, similar to innate affinity, social affinity is represented by a numerical measure.

Importantly, in real world situations, events typically come with natural cardinality constraints. For example, most sports activities need a certain number of participants: two or four for tennis, two for chess, and two to nine for poker games.

We illustrate aforementioned characteristics more concretely through the following motivating examples.

Example 1 (Convention Social Events). Academic conferences and business conventions often provide networking opportunities for attendees by offering social events. Planning them involves gathering individuals with similar interests to facilitate interactions as well as ensuring that they have a good time. The current practice is to offer these events as a "canned" list of options: e.g., everybody selects from a small set of event choices based on their interests, perhaps uses ad hoc conversations among friends for coordination. Being able to automatically assign people to events from a large list of options, in a centralized manner, based on users' interests and friendships will likely lead to people enjoying those events more.

Example 1 illustrates the core technical challenge inherent in event organiza- 
tion, viz., the assignment problem, where the system has to assign a given set of users to events, with the goal of making users happy. Other examples in this category include organization activities at a reunion party, corporate activity day, community fund raising, or volunteering gatherings. Event organization is by no means restricted to conventions, as the next example illustrates.

Example 2 (Wide Area Events). In event organization platforms like Plancast and Meetup, users across a wide area register their interests in themes or topics. Users' social information is also available, e.g., their friendships in popular social networks can be supplied to these platforms. Events are started by end users through the specification of the activity, capacity, etc. The platforms use known metadata to make ad hoc recommendations of events to users. As the platforms get utilized, information from past event attendance can be leveraged to estimate user's interest in future ones. In place of ad hoc recommendations, these platforms can make centralized assignments for all events planned in a local wide area (e.g., a city), to maximize the "social welfare" of users.

As the first systematic study towards Social Event Organization, we understand the problem on the assignment procedure illustrated by the two examples above. Specifically, we are given a set of users, who are socially connected and have inherent interests in the events being offered. We need to assign users to events so as to maximize the "overall happiness" of the users, while respecting the event cardinality constraints (minimum and maximum). In event organization over a wide area, in addition to cardinality constraints, time and location also play a role. E.g., user's proximity to the event's location may affect the user's innate affinity for the event. A similar remark holds for the time at which the event is organized. In order to allow us to focus on the key technical problem of assignment, we assume that users' proximity to the location of events as well as availability of users for the events are factored in while determining the innate affinity of a user to an event. Consequently, our focus is squarely on finding good assignments of users to events as opposed to scheduling of events.

One critical aspect of the solution is to define the overall happiness as a combination innate and social affinities. We provide an intuitive definition of social welfare, which is the sum of the total innate affinities enjoyed by the users to the 
events they are assigned, and the aggregate social affinities enjoyed by pairs of users who are assigned to the same event. The exact definition will be provided later. This focus allows us to cast the Social Event Organization problem as a constrained discrete optimization problem, for which we can explore algorithmic solutions.

Our event organization problem has close connections with several major bodies of work generalized assignment problem (GAP) [8, 9, 15, 25], a family of two-sided matching problems including the National Resident Matching Problem (NRMP) [24], and community-search problems [26]. We depart from them by taking both innate and social affinities into account and solving the event organization problem under a unified framework. A detailed comparison with these related works appears in Chapter 2.

Our event organization problem has close connections with two major bodies of work-generalized assignment problem (GAP) and a family of two-sided matching problems including the so-called college admission problem and the National Resident Matching Problem (NRMP). While a detailed comparison with this and other related work appears in , we note here that in all these problems social affinity is not a concern. Taking social affinity into account raises a significant computational challenge as we shall show.

Specifically, we make the following contributions.

- We formally define social event organization as a constrained discrete optimization problem that asks for an assignment with maximum social welfare while respecting cardinality constraints of events (Chapter 3.1).

- The problem is shown to be NP-hard. Given this, we analyze its approximability: we establish close connections between restricted versions of our problem and the SUBSET-SUM problem and DENSE $k$-SUBGRAPH problem. We exploit these connections to offer strong evidence that the problem is hard to approximate (Chapter 3.2).

- We then develop several heuristics including a greedy hill-climbing strategy and several improvements. Our improvements are based on a notion of "phantom events" which refer to tentatively scheduled events which have yet 
to meet their lower cardinality constraint, and a look-ahead based technique (Chapter 4).

- We conduct a comprehensive experimental study on both synthetic and real data sets from Plancast, Meetup and SIGCOMM 2009. Our results show that our methods with look ahead estimation far exceed the baseline methods in the value of social welfare attained. Furthermore, our most sophisticated algorithm completes event assignments on a dataset consisting of 100,000 users and 500 events in under 17 minutes (Chapter 5).

Finally, Chapter 6 concludes the thesis and summarizes interesting directions for future work. 


\section{Chapter 2}

\section{Related work}

Our event organization framework coincides with two traditional research direction on the general problem of assigning elements to a number of sets. One of the major direction on this area is the generalized assignment problem (GAP) [8, 9, 15, 25], which generalizes knapsack and bin packing problems: there are multiple bins with capacity (maximum size) constraints, multiple objects with a size and a profit for placing an object in a bin; the problem is to find an assignment of objects to bins respecting capacity such that the total profit is maximized. In a special case, all objects have the same size and capacity is replaced by (maximum) cardinality. There are two major differences between GAP and event organization. Firstly, events typically also have lower bound constraints on their cardinality. For example, a game of Mahjong cannot be played by fewer than four people. Those lower and upper cardinality constraints must be respected by the solutions we propose. Very recently, lower bound constraints have been studied in the context of GAP [15], and the precise relationship between event organization and GAP with lower bound constraints will be made clear in Section 3.2. Secondly, social affinity is not considered in GAP, whereas it is one of the key ingredients in social event organization.

The other major research direction on the assignment problem is the National Resident Matching Problem (NRMP) and the related two-sided matching problems such as college admissions [6, 10, 11, 24], where medical school graduates are matched with medical residency programs offered by hospitals throughout the 


\begin{tabular}{l|c||r}
\hline & Tennis & Movie \\
\hline Alice & 1 & 5 \\
\hline Bob & 5.01 & 2.01 \\
\hline Charlie & 5.02 & 1.02 \\
Denny & 5.03 & 1.03 \\
Eddie & 3.01 & 3 \\
\hline
\end{tabular}

Table 2.1: Innate affinity for Example 3

United States, and subsequently adopted by other countries. The differences with event organization are three-fold. First, graduates choose residency programs as individuals, and social affinity is almost never a concern, except in rare cases where two graduates are a couple. Second, in NRMP, every graduate provides a rank order list of programs she'd like to join and and every program has its own preference list over graduates. Stability is an important concern. A matching is stable if there is not a pair of a graduate and a resident program such that they both prefer each other over the resident program or graduate they are currently assigned to. Such a pair is called a blocking pair. When stable matchings exist, they focus on finding an optimal stable matching where no graduate is worse off than she would be in any other stable matching. When stable matchings don't exist as in [6, 11], they focus on finding matchings that minimize the number of blocking pairs. While stability is similar in spirit to avoiding regrets (i.e., assigning a user to an event she does not enjoy) in event organization, the notion of "stability" in event organization is not important as events treat all users alike! Third, in contrast to the objectives in NRMP and college admissions, our goal is to maximize the social welfare (defined in Section 3.1) of the assignment, taking both innate and social affinities into account, while respecting the cardinality constraints of events.

We use a concrete example to illustrate the difference between the our social event organization (SEO) problem, the GAP problem and the NRMP problem.

Example 3 (SEO, GAP and NRMP). Imagine a scenario of 5 people deciding between 2 events, where the innate affinity between each person and each events are shown in Table 2.1, and each event, happens to have the lower bound capacity constraint of 2 and upper bound capacity constraint of 3, the social affinity between each pair of persons are 0 except good friends Alice and Bob, who have an affin- 
ity value of 10. The SEO, GAP and NRMP treats this scenario in distinct ways. Starting with the simple one, the GAP would only consider the innate affinity and capacity constraint, assigning Alice and Eddie to Movie and the others to Tennis. The NRMP is also ignorant to social affinity between Alice and Bob, creating a assignment the same as above. The above scenario is "stable" in the sense that Movie event would prefer to leave the 3 spots to Bob, Charlie, Denny since they enjoy it more than the other two, leaving Alice and Eddie to the Tennis event, would happens to prefer them over the rest 3 . Finally, the SEO problem, taking account into both the innate affinity and the social affinity between Alice and Bob, would generate an ideal assignment where Alice and Bob happily stay togther for Movie, and the rest three go the Tennis, which they would also enjoy very much, achieving an optima in a globale sense.

In Section 4, we adapt the NRMP solution to solve our problem, and empirically compare it in Section 5 .

Beyond the traditional research In a sense, event organization can be seen as being akin to group recommendations: so long as we know which set of users will attend the same event, i.e., group memberships, but not which one, we can, in principle, determine that event using group recommendations [2]. However, we do not know these groups and part of the challenge is to determine them. Besides, our assignment of users (and hence of groups) to events is more "holistic" in taking not just innate affinity, but also social affinity into account. It is their combination that drives our assignment. Thus, group recommendations cannot handle our problem.

In team formation in social networks [3, 16], they assume that for each user a set of skills is available and additionally, a measure of compatibility between pairs of users is also known. The problem is to put together teams that satisfy the skill requirements of a given task while minimizing the communication overhead of the team, e.g., the diameter or weight of the minimum spanning tree of the graph of the formed team. Variants of the problem include ones where different cost models are used and ones where the tasks arrive online and the team formation has to keep the workload of users balanced. The problem is NP-hard and many of the works resort to approximation algorithms. Superficially, the team formation problem seems similar to ours. However, while social affinity has a counterpart 
in compatibility, there is no direct innate affinity between users and tasks, except indirectly via skills. Furthermore, and more important, cardinality constraints in event organization make the problem significantly more challenging. Finally, a user may be assigned to more than one task whereas users are assigned to at most one event in our problem.

Also relevant to our work is the community search problem [26], where given a set of query nodes, the task is to find a community containing the query nodes that maximizes the overall social affinity, subject to a maximum cardinality constraint. However, there is no notion of events, nor users innate affinities towards events in [26]. In contrast, our problem involves a combination of social and innate affinities and thus a holistic approach is needed which at once pays attention to the innate aspect (matching quality) and the social aspect. There have also been studies on geo-social query processing [4]. Though both our problem and theirs consider social effects, the SEO problem is inherently an assignment problem and cannot be solved by issuing queries.

Yet another related research direction is social graph partition and more formally "Uniform Metric Labeling"[5, 14], which is an optimization problem that assigns group labels to nodes in the graph to minimize, incorporating both the "innate affinity", the cost of assigning specific labels to nodes, and the "social affinity", the cost of assigning friends to different groups. Similarly to the team formation problem, social graph partition does not consider cardinality constraints in event organization, which is one of central element of hardness results and algorithm design. 


\section{Chapter 3}

\section{Social event organization}

In this chapter, we formulate the assignment variant of the Social Event Organization problem, the focus of our research, and study its hardness and approximation.

\subsection{Problem definition}

Consider a setting where organizers of a large gathering (e.g., an international conference or a company-wide retreat) are planning social activities for the conference attendees. Or a scenario in which or an event planning platform (e.g., Meetup) is being used to plan social events for its users over a wide local area such as a city. In both cases, there is a set of users $U$ who must be assigned to events or activities from a given set of possible events $A$. We assume the presence of a social network, through which the friendships among those users can be obtained. Let $G=(U, E)$ be the friendship graph induced on $U$. Each event $a \in A$ is associated with a minimum cardinality bound $\gamma_{a}$ and a maximum cardinality bound $\delta_{a}$, as motivated in Section 1 .

There is a function $\sigma: U \times A \rightarrow \mathbb{R}_{+}$such that for each user $u$ and event $a$, $\sigma(u, a)$ models the user's innate affinity for the event. We often denote $\sigma(u, a)$ as $\sigma_{u, a}$ for simplicity. As mentioned in the introduction, we assume that the innate affinity $\sigma_{u, a}$ factors in $u$ 's proximity to $a$ 's location and $u$ 's availability w.r.t. the time at which $a$ is held. In addition to their innate affinity to events, users have pairwise social affinity with each other, and we capture this by a function $w: U \times U \rightarrow$ $\mathbb{R}_{+}$, such that $w(u, v)$ is the social affinity between $u$ and $v$. Both innate affinity 
to events and social affinity to other users attending the same event contribute to a user's sense of "utility" for attending an event. Note that as discussed in Section 1 , social affinity is important because an individual may enjoy an event more when she is joined by her friends. We assume in this paper that social affinity is nonnegative and that social affinity values are symmetric, i.e., $w(u, v)=w(v, u)$, for all $u, v \in U$.

Suppose a set of users $S \subset U$ is assigned to an event $a \in A$. We define the utility of this assignment as:

$$
\mu(S, a)=\sum_{u \in S} \sigma_{u, a}+\sum_{u, v \in S, u \neq v} w(u, v)
$$

That is, the overall utility of the set of participants of an event is the sum of the total innate affinity of the participants for the event and the total social affinity of all pairs of participants. As a special case, we define $\mu(\emptyset, a)=0$ : when nobody is assigned to an event, it results in zero utility. As mentioned in the introduction, both innate affinity $\sigma_{u, a}$ and social affinity $w(u, v)$ can be provided by the users explicitly through stated interests and friendships. However, in case such explicit information is not available, those values can be estimated from a user's implicit interests and past activities. For example, social affinity can be computed using well-studied social distance measures (e.g., graph distance, Katz, or hitting time) [18]. We defer the details to Section 5 .

An assignment is a (possibly partial) function $M: U \rightarrow A$. For $a \in A$, we denote by $M^{-1}(a)$ the (possibly empty) subset of users assigned to $a$, i.e., $M^{-1}(a)=$ $\{u \in U \mid M(u)=a\}$. An assignment $M$ is said to be feasible provided all cardinality constraints are satisfied, i.e., $\forall a \in A$ for which $M^{-1}(a) \neq \emptyset$, we have $\gamma_{a} \leq\left|M^{-1}(a)\right| \leq \delta_{a}$, in words, for all events to which at least one user is assigned, the number of users assigned to that event lies within its lower and upper bounds. Notice that owing to the cardinality constraints and the number of users and events in an instance of the assignment problem, it may not be possible to find a feasible assignment that covers all users: e.g., we may have 10 users and 1 event such that the event can accommodate at most 5 people. This is the reason we allow partial functions above. Similarly, not all events may be scheduled by an assignment, i.e., $M$ need not be onto. We define the social welfare of a feasible assignment $M$ as 


$$
\omega(M)=\sum_{a \in A} \mu\left(M^{-1}(a), a\right)
$$

The overall social welfare of an assignment is thus determined by the sum of utilities of the assignments made to each event. Our objective is to find a feasible assignment that maximizes the overall social welfare. Our definition of overall social welfare reflects the following intuition. A user's personal utility for an assignment increases with her innate affinity for the event, as well as the social affinity she has toward other users attending the same event. Besides, the more such fellow attendees, the higher her utility. The definition of overall social welfare is a simple extension of this intuition. Note that other definitions of social welfare, using other aggregate functions, are possible: we prefer to use a simple definition here that captures the intuition above. We formally define the Social Event Organization (SEO) problem as follows.

Problem 1 (Social Event Organization (SEO)). Given a set $U$ of users and the induced social graph $G=(U, E)$, a set $A$ of events where each $a \in A$ has a minimum and maximum cardinality bound, denoted $\gamma_{a} \in \mathbb{N}$ and $\delta_{a} \in \mathbb{N}$ respectively $\left(\gamma_{a} \leq\right.$ $\delta_{a}$ ), innnate affinity function $\sigma(\cdot, \cdot)$, and social affinity function $w(\cdot, \cdot)$, produce a feasible assignment of $M: U \rightarrow A$ that has the maximum overall social welfare $\omega(M)$, i.e., find

$$
M^{*}=\arg \max \{\omega(M) \mid M \text { is feasible }\} .
$$

The number of scheduled events (i.e., those have at least one user assigned and cardinality constraints satisfied) is not fixed in advance, but rather determined by the solution, in which some events may receive zero users. However, the cardinality constraints for each event that has non-zero participants assigned must be respected. It is possible to have multiple groups performing the same event, e.g., there may be four groups of two participants each that are suggested the event of playing chess. This is easily handled by technically treating different instances of a given event as different events. We can consider weighting the two terms in Eq. 3.1, corresponding to the innate and social contributions to social welfare, differently. Our results in the next section show that the problem remains hard 
regardless of the weights chosen.

\subsection{Hardness results}

Not surprisingly, our first result is that Social Event Organization is NP-hard. Our reduction is from the recently studied Seminar Assignment Problem (SAP). SAP is obtained by adding lower bound constraints to a restricted version of GAP where all objects have the same size, and is formally stated as follows. Given a set of $n$ students and and a set of $m$ seminars with maximum cardinality $B_{1}, B_{2}, \ldots, B_{m} \in \mathbb{N}$ and minimum cardinality $q_{1}, q_{2}, \ldots, q_{m} \in \mathbb{N}, q_{i} \leq B_{i}$, a profit $p_{i, j} \in \mathbb{N}$ by assigning student $i$ to seminar $j$, we want to find an assignment of students to seminars that satisfies the cardinality constraints and maximizes the total profit. Krumke and

Thielen [15] recently showed that this problem is NP-hard. We have the following easy result:

Theorem 1. The decision version of Social Event Organization is NP-complete.

Proof. Clearly, SAP is isomorphic to a restricted version of SEO where all innate affinities are natural numbers and all social affinities are zero. The NP-hardness of SEO follows from this. Membership in NP is straightforward: given an assignment, both checking its feasibility and whether its social welfare exceeds a given threshold can be done in polynomial time.

Next, we analyze how hard it is to approximate SEO. Our analysis involves two special cases of the problem, one with only innate affinities (which is isomorphic to the SAP problem) and the other with only social affinity.

Special Case 1: Innate Utility Only. In this case, we only consider problem instances of which $w(u, v)=0$, for all users $u$ and $v$. Thus, the problem is to find a feasible assignment $M: U \rightarrow A$ that maximizes the social welfare, which is solely determined by innate affinities. We call this restricted version SEO-Innate.

Theorem 2. It is NP-hard to approximate SEO-Innate within a factor of $(1-1 / n+$ $\varepsilon)(\forall \varepsilon>0)$ in polynomial time, where $n$ is the number of users.

Proof. We prove the result by showing that if such an algorithm existed, it could solve the SUBSET-SUM problem, an NP-complete problem, in polynomial time. 
Since this is impossible unless $\mathrm{P}=\mathrm{NP}$, the theorem follows. Consider an arbitrary instance $\mathscr{I}$ of SUBSET-SUM consisting of a set of integers $T=\left\{t_{1}, t_{2}, \ldots, t_{N}\right\}$ and a target number $\tau$. We must find out if there is a subset of $T$ whose elements sum to exactly $\tau$. Algorithm $\mathscr{B}$ first creates an instance $\mathscr{J}$ of the SEO problem with $\tau$ users and $N$ events, and for each event $a \in[1, N]$, it sets $\gamma_{a}=\delta_{a}:=t_{a}$. Also, $\sigma_{u, a}=1$ for all $u \in U, a \in A$ and $w(u, v)=0$ for all $u, v \in U$. Then $\mathscr{B}$ runs $\mathscr{A}$ on $\mathscr{J}$, and outputs YES (indicating there is a subset of $T$ summing to exactly $\tau$ ) if and only if $\mathscr{A}$ outputs an assignment with social welfare $\tau$. We next prove that algorithm $\mathscr{B}$ can correctly distinguish between the YES- and NO-instances of SUBSET-SUM.

If $\mathscr{I}$ is a YES-instance, then by the above reduction, $O P T_{\mathscr{J}}=\tau$, i.e., the maximum possible social welfare of any feasible assingment on instance $\mathscr{J}$ is $\tau$. By assumption, $\mathscr{A}$ will output an assignment with welfare $\geq(1-1 / \tau+\varepsilon) \tau>$ $\tau-1$. Since all utility values are 1 , the output of $\mathscr{A}$ will be exactly $\tau$, and thus $\mathscr{B}$ answers correctly: YES.

If $\mathscr{I}$ is a NO-instance, then for $\mathscr{J}$, by construction, not all users can be fit into an event, implying $O P T_{\mathscr{J}}<\tau$. Since the output of $\mathscr{A}$ is always $\leq O P T_{\mathscr{J}}$, it will be surely $<\tau$. Again, algorithm $\mathscr{B}$ answers correctly: NO. This was to be shown.

We note that complementarily, Krumke and Thielen [15] show that it is NPhard to approximate SAP within a factor of $1-\varepsilon_{0} / 3$ even when all profits are in $\{0,1\}$, where $\varepsilon_{0}>0$ is a constant associated with the hardness gap of the 3Bounded 3-Dimensional Matching problem [21]. As a consequence, they show that SAP does not admit PTAS (polynomial time approximation scheme). This hardness is clearly inherited by SEO-Innate as well. Note that both $1-\varepsilon_{0} / 3$ and $(1-1 / n)$ are close to 1 (the latter when $n$ is large). This raises the question whether coarser approximations exist for SEO. Our result in the next section suggests it is unlikely.

Special Case 2: Pair-wise Affinity Only. In this case, we restrict attention to instances of SEO where $\sigma_{u, a}=0$ for all users and all events. We refer to this restricted version of SEO as SEO-Social. We show that this restricted problem is hard to approximate within any constant factor under hardness assumptions about certain combinatorial problems. Specifically, our result is achieved by a reduction 
from the DENSE $k$-SUBGRAPH problem. That problem, given a graph $G=(V, E)$ and a parameter $k$, asks to find a subgraph $G^{\prime}$ of $G$ induced by $k$ nodes such that average degree of nodes in $G^{\prime}$ is maximum. The average degree of a node in $G^{\prime}=$ $\left(V^{\prime}, E^{\prime}\right)$ is given by $2\left|E^{\prime}\right| /\left|V^{\prime}\right|$. By assuming the hardness of a conjecture known as Unique Games with Small Set Expansion Conjecture [13], Raghavendra and Streurer [23] show that DENSE $k$-SUBGRAPH is hard to approximate within any constant factor. We "lift" this to show a similar hardness of approximation of SEOSocial. For lack of space, we refer the reader to [23] for details and history of this conjecture.

Theorem 3. Assuming the Unique Games with Small Set Expansion Conjecture, it is NP-hard to approximate SEO-Social within any constant factor in polynomial time.

Proof. Given an instance $\mathscr{I}$ of DENSE $k$-SUBGRAPH defined by $G=(V, E)$ and a positive integer $k$, create an SEO instance $\mathscr{J}$ by setting $A=\{a\}, \gamma_{a}=\delta_{a}=k$, and $U=V$. That is, there is just one event whose lower and upper bound on cardinality is $k$ and there is a user corresponding to each node of $G$. For all $u \in U$, set $\sigma_{u, a}=0$, and for all $u, v \in U, w(u, v)=1$. By construction, $O P T_{\mathscr{I}}=O P T_{\mathscr{J}}$, that is, the maximum social welfare of a feasible assignment on instance $\mathscr{J}$ is identical to the maximum average degree of a $k$-vertex induced subgraph of $G$ in instance $\mathscr{I}$. Suppose there is a polynomial-time algorithm $\mathscr{A}$ approximating SEO within a factor of $c \in(0,1)$. Then, $\mathscr{B}$ can approximate DENSE $k$-SUBGRAPH within the same factor by converting $\mathscr{I}$ to $\mathscr{J}$ as above, running $\mathscr{A}$ on $\mathscr{J}$, and outputting the nodes corresponding to the users chosen by $\mathscr{A}$ to attend event $a$. This is not possible unless the Unique Games with Small Set Expansion Conjecture does not hold.

To conclude this section, not only is SEO NP-complete, it is also made up of two hard subproblems. Our results show that it is unlikely to be approximable within any constant factor in polynomial time, unless some hardness assumptions about important combinatorial problems break down. By Theorems 2 and 3, this statement is obvious regardless of the weights chosen for the two terms in Eq. 3.1. 


\section{Chapter 4}

\section{Proposed solutions}

Given that SEO is NP-hard to even approximate, we propose a variety of heuristics that give emphasis to different aspects of the problem, such as the social affinity, the innate affinity, and the cardinality constraints. Before diving into the details of the algorithms, it is important to first understand what constitutes a feasible (valid) solution to the SEO problem.

Characterizing Feasible Solutions. For any event, if the event has reached its minimum cardinality, we refer to it as a real event; otherwise, we call it a phantom event. For example, a tennis or chess game with only one player is a phantom event, that will become real only after one other person is assigned to the event. An event is open and can accept more participants if the maximum cardinality has not been reached yet, otherwise it is declared closed. By definition, all phantom events are open. However, a real event can be either open or closed. A user $u$ is available if $u$ has not been assigned to a real event yet. A user-event assignment is valid if it involves an available user and an open event. During the assignment process, if the event to which a user was assigned becomes real, then the user's assignment is fixed, and she is marked unavailable. Consequently, all other previous and future assignments involving that particular user are deemed invalid. Recall that the final solution must be feasible, i.e., respect cardinality constraints. As shown in Section 3, in some instances it is not possible to find an assignment for every user, so the assignment function may be partial. In practice, we can deal with this by supposing that there are sufficiently many events with large capacity such 
as movies or theatrical shows to which users left unassigned by the algorithm may be assigned.

Notice that each assignment decision impacts the utility received from future assignments, due to the coupling effect with other users in the form of social affinity. Therefore, it is non-trivial to achieve a (feasible) solution to SEO that achieves a high social welfare.

Solution Template. In the remainder of this chapter we discuss a variety of algorithms. At a high level, each algorithm (except Random and NRMP+) follows the following template. First, a sorted list $\mathscr{L}$ of potential assignments of users to events is generated. The additional contents of the list may vary depending on the algorithm. This list may be updated and re-ordered several times during a run of the algorithm. Second, users are assigned to events by making one pass on this list, and the "state" of users (available, unavailable), and events (phantom, real open, real closed) is updated appropriately. In particular, it marks an event $a$ as "real" when it reaches its minimum cardinality $\gamma_{a}$, and "closed" when it reaches its maximum cardinality $\delta_{a}$. These assignments are tentative and are recorded by membership in the " $S$ " sets, e.g., $u \in S_{a}$ means $u$ is tentatively assigned to event $a$, and possibly to other events. All events are "open" and "phantom" to start with. Once an event $a$ becomes real, the users in the set $S_{a}$ are marked "unavailable" for future assignments and we set $M(u)=a, \forall u \in S_{a}$. Additionally, the algorithm invalidates any previous and future assignments of each user $u \in M^{-1}(a)$, by cleaning up other " $S$ " sets as needed. Finally, the algorithm performs post-processing to ensure that the output is a feasible solution, i.e., no phantom events are left behind.

\subsection{Baselines}

In this section, we present a set of intuitive baseline solutions, in increasing level of sophistication. We build on these baselines and present our proposed algorithms in Section 4.2 .

\section{Random}

Our first baseline is called Random, which randomly assigns users to events while respecting the cardinality constraints. Specifically, the algorithm first shuffles the 


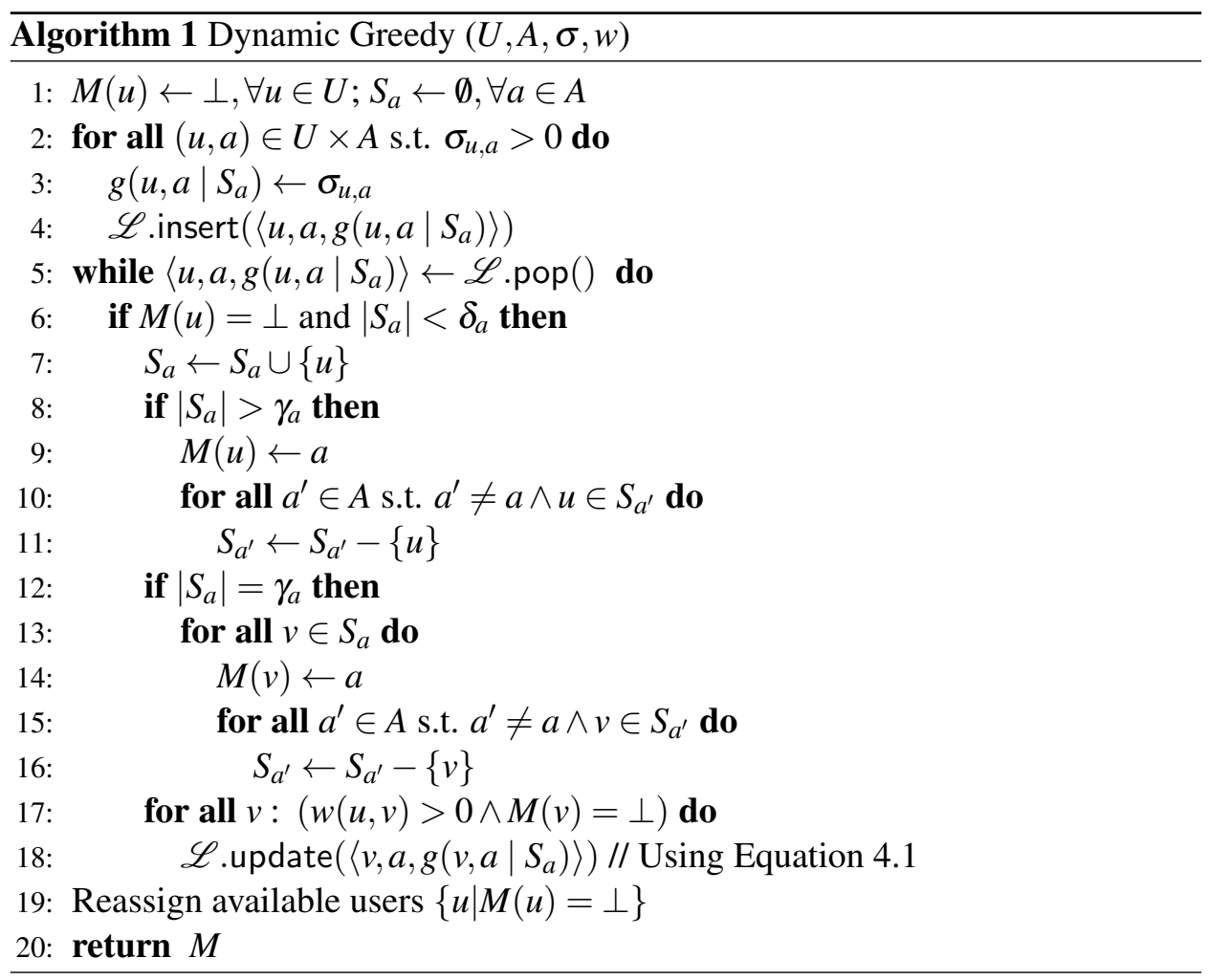

list $\mathscr{L}$ containing tuples $\langle u, a\rangle$ of user-event pairs randomly. It then traverses the list and at each tuple $\langle u, a\rangle$ assigns user $u$ to event $a$ if $u$ is available and $a$ is open. It appropriately marks an event as real when it reaches its minimum cardinality constraint. Clearly, this is a "straw man" approach, which does not take into account the "utility" of any assignment.

\section{NRMP+}

Given the connection between SEO and NRMP, a natural question is whether we can leverage algorithms developed for NRMP. We next describe an adaptation of the NRMP algorithm [10] for handling the lower bound constraints present in SEO. In this adaptation, called NRMP+, we cast SEO in the NRMP framework by associating a preference list of events for each user, ordered by the innate affinity, and 
a preference list of users for each event, ordered by the innate affinity. ${ }^{1}$

NRMP+ repeats the following procedure until each user is either "accepted" by an event or is "rejected" by all events she applied to. Initially, all users are unassigned and no acceptance or rejection has been made yet. In each round, each unassigned user "applies" to the most preferred event that has not rejected her. Each event $a$ then picks the $\delta_{a}$ most preferred users who applied to it, or all applicants if there are fewer than $\delta_{a}$ of them, and puts them on a waiting list. Users who failed to get on the waiting list are rejected and are thus unassigned after this round. After this iterative process stops, we check if there exists any phantom event. If yes, redistribute users assigned to phantom events to existing real open events in a greedy manner, based on innate affinity. After that, either no user is left unassigned or there are no real events to which unassigned users can be assigned, and we stop.

It is unclear how NRMP can be directly adapted to take social affinity into account in deciding acceptances. In Section 5, we empirically compare NRMP+ with the more sophisticated baselines we develop next, as well as with our main algorithm.

\section{Static Pairwise Greedy}

Our next baseline, called Static Pairwise Greedy (SG), assigns pairs of users to events, taking into account both innate event and pairwise social affinities. Specifically, a sorted list $\mathscr{L}$ containing tuples $\langle u, v, a\rangle$ representing potential assignment of pairs of users to events is generated. The list is ordered by non-increasing potential gain (of "utility"), defined as

$$
g((u, v), a)=\sigma_{u, a}+\sigma_{v, a}+2 \cdot w(u, v) .
$$

The list is traversed and at each tuple $\langle u, v, a\rangle$ users $u$ and $v$ are assigned to event $a$ if both users are available, and $a$ is open with at least two spots. User and event states are appropriately updated. Finally, any users that are in phantom events at the end of one pass over the list $\mathscr{L}$ are redistributed greedily among remaining open events. We call this a static approach as the function $g$ is computed only

\footnotetext{
${ }^{1}$ Events don't have independent preference lists: we do this for the sake of simulation of NRMP.
} 
once. Therefore, the partial ordering of the $\mathscr{L}$ is static, and it only incurs deletions (with some assignments being invalidated). Therefore, the assignment of a pair of users to an event is oblivious to which users are already assigned to that event.

\section{Dynamic Greedy}

Our next algorithm, called Dynamic Greedy (DG), is more sophisticated. It updates its estimation of the "utility" a user will attain from an assignment, based on the current set of assignments. To achieve this, the algorithm updates the list $\mathscr{L}$ at each assignment, where $\mathscr{L}$ is composed of tuples $\left\langle u, a, g\left(u, a \mid S_{a}\right)\right\rangle, S_{a}=\emptyset$ initially, and the potential gain $g$ is

$$
g\left(u, a \mid S_{a}\right)=\sigma_{u, a}+\sum_{v \in S_{a}} w(u, v)
$$

Clearly, when no users are assigned to an event $a, g(u, a \mid \emptyset)=\sigma_{u, a}$. Algorithm 1 presents the pseudocode for the DG method. At each step, the assignment that gives the largest potential gain $g($.$) is performed. Let N_{u}$ be the set of users $v$ : $w_{u, v}>0$. Now, whenever a user $u$ is assigned to an event $a$, we need to recompute the potential gain $g\left(v, a \mid S_{a}\right)$ for all neighbors $v \in N_{u}$ of user $u$ in the network, since their utility for event $a$ increases after the assignment of $u$ to that event. In practice, the algorithm maintains the list $\mathscr{L}$ as a priority queue of tuples in non-increasing order of $g\left(u, a \mid S_{a}\right)$. We use the notation $M(u)=\perp$ to denote that the user $u$ is available. We remark that the update operation (line 18) is in charge of not only updating the existing entries in the priority queue but also checking whether the pair $(v, a)$ is already in the heap and if not, adding it.

The post-processing in Line 19 is done in a greedy manner. Let $A^{\prime}$ be the set of currently open real events, i.e., $A^{\prime}=\left\{a\left|\gamma_{a} \leq\right| S_{a} \mid<\delta_{a}\right\}$ and $U^{\prime}$ be the set of users assigned to phantom events. The list $\mathscr{L}$ is re-constructed with users from $U^{\prime}$ and events from $A^{\prime}$, and we rerun the DG algorithm over this list. This process can be repeated as needed. The post-processing stops when either all users are assigned or there are not enough real events (i.e., all real events are closed). In the latter case, from a practical perspective, the remaining users may be assigned to new events with a large capacity, e.g., watching a movie or a theatrical show. 
Implementation Notes and Time and Space Complexity. We remark that in our implementation, we optimize the algorithm for better performance. In particular, we tune our implementation to suit a Fibonacci heap based realization of the priority queue. Specifically, we commit to making an assignment for new users right after line 7. This allows us to avoid assigning users to multiple phantom events. Thus, when the priority queue is updated, "decrease key" operation will not be needed, permitting us to implement the priority queue using a Fibonacci heap. We note, however, in this case the assignment function $M$ no longer corresponds to only real events since some phantom events may be left behind by the algorithm at termination. For ease of exposition, we have chosen to present our algorithm in a conceptually simpler way.

When the priority queue $\mathscr{L}$ is implemented using a Fibonacci heap, then for each assignment $(u, a)$, the algorithms performs $\mathrm{O}\left(\left|N_{u}\right|\right)$ update operations (Line 18), each of which is constant time. Summing over all users, the total running time of all update operations is $\mathrm{O}(|E|)$, where $E$ is the edge set of the social network of users, or more precisely the set of pairs $(u, v)$ with $w_{u v}>0$. Next, since the number of all possible $(u, a)$ pairs is bounded by $|\mathscr{L}|$, we need at most that many pop operations, which in total takes $\mathrm{O}(|\mathscr{L}| \log (|\mathscr{L}|))$ time. In addition, insertion is constant time for Fibonacci heaps so the initialization step takes $\mathrm{O}(|\mathscr{L}|)$. Hence, the total time complexity for Dynamic Greedy is $\mathrm{O}(|\mathscr{L}| \log (|\mathscr{L}|)+|E|)$. Finally, the space complexity is $\Omega(|\mathscr{L}|+|E|)$.

\subsection{Greedy with look ahead estimation}

The Dynamic Greedy algorithm is intuitive: it tries to make assignments based on the largest estimated gain, given the current state of the assignment. However, it has its limitations: (i) it is oblivious to which events a user's friends are likely to be assigned; (ii) the first pass may result in a large number of phantom events as it is ignorant of which events are likely to materialize. To address these limitations, we propose the Phantom- and Community-Aware Dynamic Greedy or PCADG. A key feature of this algorithm is that it looks ahead at the forthcoming assignments to inform the decision at each step.

Community Awareness. The earlier algorithms do not take into account the po- 


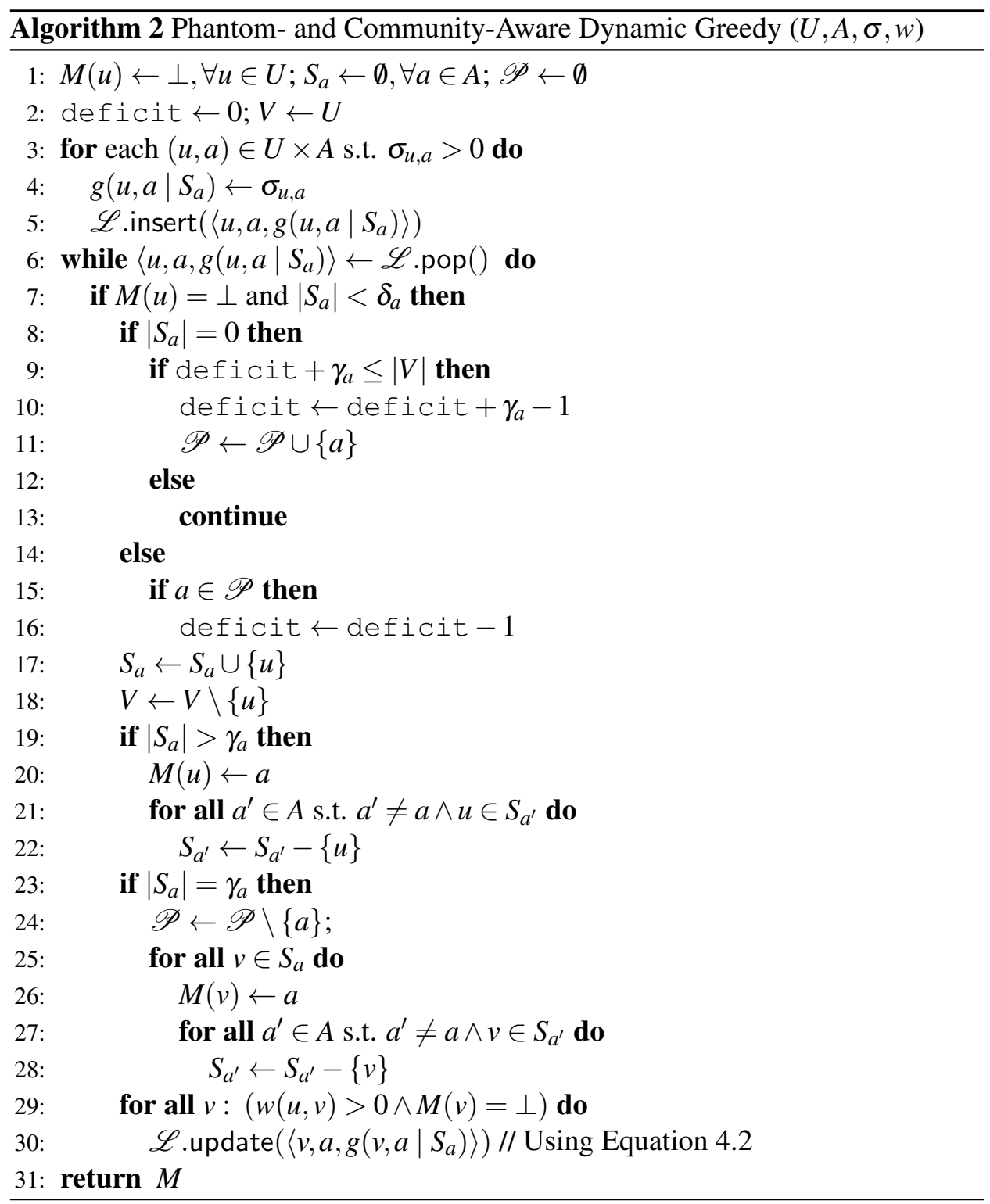

tential gain that future assignments of friends to an event may bring to a user. In our Phantom- and Community-Aware Dynamic Greedy algorithm, the awareness of friends' potential assignments is incorporated into the decision making process. 
More specifically, here, the potential gain $g$ is defined as

$$
\begin{aligned}
g\left(u, a \mid S_{a}\right)= & \sigma_{u, a}+\sum_{v \in S_{a}} w(u, v) \\
& +\left(\delta_{a}-\left|S_{a}\right|\right) \sum_{v \in V} w(u, v) /(|V|-1) .
\end{aligned}
$$

Notice that the first two terms in this equation are identical to the two terms in Equation 4.1 of the Dynamic Greedy method. The third term corresponds to the "look-ahead" whereby it is optimistically assumed that the remaining spots in the event $a$ will be filled with friends of $u$, with an average social affinity toward $u$. The average is computed over users who are not assigned to any event yet. While the first two terms are based on the current membership of $S_{a}$, the third term is based on anticipation. Thus, this algorithm gives equal importance to both innate affinity and social affinity in gauging the potential gain that could come from an assignment. Other variants can be easily designed to weigh the importance of the innate and social affinities differently.

Phantom Awareness. Another observation about Dynamic Greedy is that it tends to be somewhat indiscriminate in the creation of phantom events: it never restricts the creation of new events as the condition $\left|S_{a}\right|<\delta_{a}$ (line 6 of Algorithm 1) will always be true for new events, for which $\left|S_{a}\right|=0$ by definition. All phantom events that are left behind after a pass over the list $\mathscr{L}$ have to be cleaned up later. PCADG tries to limit the creation of phantom events, by employing a stronger condition for phantom event creation. Accordingly, it keeps track of phantom events as it proceeds, and procrastinates on creating new events if the number of unassigned users is less than the currently unfilled spots in existing phantom events that need to be filled in order for the events to materialize. To this end, the algorithm maintains a variable called deficit, computed as,

$$
\text { deficit }=\sum_{a \in \mathscr{P}}\left(\gamma_{a}-\left|S_{a}\right|\right)
$$

where $\mathscr{P}$ is the set of phantom events and $S_{a}$ is the set of users assigned to event $a$. In addition, the algorithm maintains the set of users $V$ that have not yet been 
assigned to any event, phantom or real. Specifically,

$$
V=\left\{u \mid u \in U \wedge M(u)=\perp \wedge u \notin \bigcup_{a \in A} S_{a}\right\}
$$

The pseudocode for Phantom- and Community-Aware Dynamic Greedy is presented in Algorithm 2. At the beginning of the algorithm, when $\mathscr{P}=\emptyset$, deficit $=$ 0 and $V=U$. The size of $V$ is non-increasing throughout the algorithm. For any assignment being considered $\left\langle u, a, g\left(u, a \mid S_{a}\right)\right\rangle$ for an available user $u$ and open event $a$, if $a$ is a new event with no users, i.e., if $\left|S_{a}\right|=0$, deficit is incremented by $\gamma_{a}-1$, else deficit is decremented by 1 . Furthermore, $u$ is removed from $V$, if $u \in V$ before this assignment. Using deficit and $V$, the algorithm limits the creation of new phantom events. If deficit $>|V|$, there will be at least one phantom event at the end of one pass over $\mathscr{L}$, thus requiring reassignment. The algorithm performs an assignment based on a list tuple $\langle u, a, g()$.$\rangle only if it does$ not push deficit over $|V|$ (Line 9). If the condition is not met, it continues to the next tuple from the list $\mathscr{L}$.

It is worth noting that PCADG may consider user-event combinations for which the innate affinity may be zero, as this assignment may potentially result in a large gain owing to the social affinity with other users assigned to this event, as per Equation 4.2. This is in keeping with our stated objective of maximizing social welfare. Therefore, it is possible that some users may be assigned to low innate affinity events as a result. We measure this tradeoff empirically in Section 5 by measuring the "regret ratio" (defined in Section 5) of the assignment returned by the various algorithms.

In order to explicitly study the impact of community and phantom awareness, we will compare PCADG with a lesser variant called Phantom-Aware Dynamic Greedy (PADG) that only takes into account phantom awareness. As such, the PADG algorithm is identical to Algorithm 2, with the one change that the potential gain is defined using Equation 4.1 instead of Equation 4.2. In Section 5, we compare the running times and scalability of different proposed algorithms, in addition to comparing them on their quality.

We close this section by noting that the time and space complexity of the PCADG algorithm is the same as that of DG (and PADG) except that the size 
of the list $\mathscr{L}$ may potentially become $O(|U| \times|A|)$ in the worst case. Also, while DG may incur multiple iterations owing to redistribution, the two algorithms have the same worst case time complexity. 


\section{Chapter 5}

\section{Experimental analysis}

We evaluate our Phantom- and Community-Aware Dynamic Greedy (PCADG) method against Phantom-Aware variant (PADG) and the baselines described in Section 4.1, i.e., Random, NRMP+, Static Greedy (SG) and Dynamic Greedy (DG).

Evaluation Metrics. The most natural evaluation metric is the total social welfare, $\omega(M)$ defined in Equation (3.2). In addition, since optimal or near-optimal solution cannot be found in polynomial time, we measure the effectiveness of each algorithm "indirectly" by comparing the utility a user gets against a coarse upper bound, which is the maximum utility she could have enjoyed by going to her favorite event with her best friends. Formally, we define the regret ratio for a user $u$, denoted $\rho(u)$, to be

$$
\rho(u)=1-\frac{\sigma_{u, M(u)}+\sum_{v \in S_{M(u)}} w(u, v)}{\max _{a \in A}\left(\sigma_{u, a}+\sum_{v \in B_{u}} w(u, v)\right)},
$$

where $M$ is the assignment made by an algorithm, and $B_{u} \subseteq N_{u}$ is the set of top- $K_{u, a}$ friends of $u$ in terms of social affinity $w(u, v)$, and $K_{u, a}=\min \left\{\left|N_{u}\right|, \delta_{a}-1\right\}$.

Ideally an algorithm that performs well w.r.t. this metric should assign users to events in such a way that many users experience low regret in the assignment made. Note that $\sum_{u \in U} \max _{a \in A}\left(\sigma_{u, a}+\sum_{v \in B_{u}} w(u, v)\right)$ is guaranteed to be greater than the optimal social welfare, except when the favorite event of all users are the same, in which case the optimal welfare reaches this quantity. Moreover, $\rho$ is a very 
pessimistic ratio, as the denominator is an upper bound on the maximum possible utility that the user can achieve. Also, it is worth pointing out that our algorithms are not designed to directly minimize individual regrets of users, but rather social welfare that is the overall utility enjoyed by all users. The purpose here is to gain some insights about to what extent our algorithms would lead to a compromise w.r.t. regret.
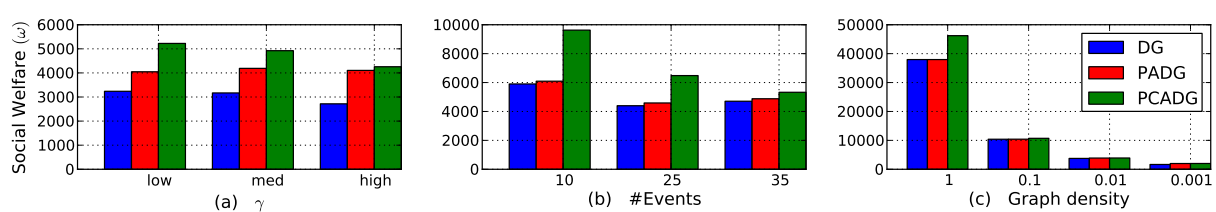

Figure 5.1: Effects of parameter choices on the performance of PADG and PCADG

\subsection{Experiments on synthetic datasets}

First, to better understand the impact of various parameters on our proposed algorithms, we first perform rigorous evaluation on synthetically generated data. We also conduct scalability tests on large synthetic data to demonstrate that our algorithms are scalable and efficient.

For all events $a \in A, \delta_{a}$ is sampled from a normal distribution with mean 20 and variance 10 , and given that, $\gamma_{a}$ is sampled uniformly at random from the interval $\left[1, \delta_{a}\right]$. The social network $G=(U, E)$ is generated by a random power-law graph model with a power-law exponent of 1.5 [1]. The data generated has 500 users and 50 events. A user is interested in an event with probability 0.05 , and thus the average number of events he is interested is 2.5 . For each $(u, v) \in E$, the social affinity value is sampled from a normal distribution with mean 1.5 and variance 3 , so are innate utilities $\sigma_{u, a}$ for all $(u, a)$ pairs in the user-event relation. All values less than $10^{-5}$ are set to be zero, and hence all affinity values are non-negative. This gives the default setting for the parameters used to generate the data, however, throughout this section, we vary these parameters to understand their effect on the performance of our three dynamic greedy methods, DG, PADG and PCADG. Figure 5.1 shows the social welfare achieved by these algorithms with three types of 
comparisons: minimum cardinality constraints, total number of events, and density of the social network graph.

Effect of Minimum Cardinality. The first test is to alter minimum cardinality constraint $(\gamma)$ and evaluate its effect on PADG and PCADG. We keep the maximum cardinality constraint $\delta_{a}$ s unchanged from the basic setting for each event $a \in A$, and vary their $\gamma_{a}$ s. We generate three groups of data, by sampling the minimum cardinality constraint $\gamma_{a}$ uniformly at random from $\left[\delta_{a} / 2, \delta_{a}\right]$ (low mean), $\left[\delta_{a}-\right.$ $\left.\delta_{a} / 4, \delta_{a}\right]$ (medium mean), and $\left[\delta_{a}-\delta_{a} / 8, \delta_{a}\right]$ (high mean) respectively. As seen in Figure 5.1(a), there is a noticeable improvement in social welfare for PADG and PCADG over DG (which does not account for phantom events). As $\gamma$ increases, more phantom events may be created, which increases the gain of PADG methods over DG.

Effect of the Number of Events. In the second test, we vary the number of events as 10, 25 and 35, for a 500 user dataset. Figure 5.1(b), shows the social welfare of the three dynamic greedy algorithms. As the number of events increases, each event needs to accommodate fewer users and thus the effect of social affinity decreases. Clearly, with fewer events, PCADG is at an advantage over PADG and DG, as it is community aware and takes into account social affinity values by looking ahead.

Effect of Graph Density. Next, we test various graph densities of the underlying social network graph by generating four different graphs using Erdős-Rényi model $G(500, p)$ where the edge existence probability being $p=0.001,0.01,0.1$, and 1 respectively. For denser graphs, more social affinity values are present and hence we observe that the margin between PCADG and the rest two is higher (Figure $5.1(\mathrm{c})$ ) in comparison with sparse graphs.

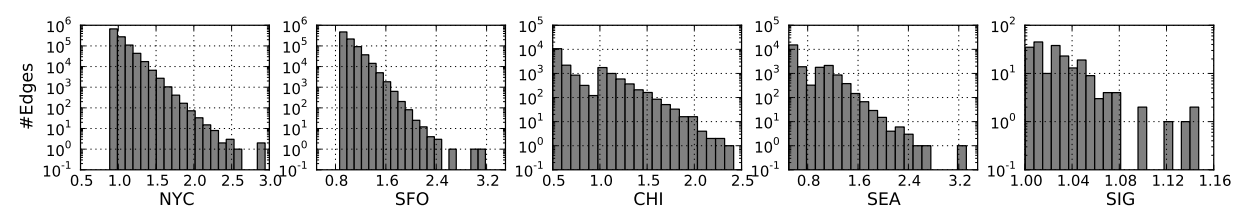

Figure 5.2: Distributions of social affinity values 


\begin{tabular}{|l|l|l|l|l|l|l|}
\hline Algorithm & $|U|$ & \multicolumn{5}{|c|}{ Number of Events } \\
\cline { 3 - 7 } & & 50 & 100 & 150 & 200 & 500 \\
\hline PADG & $1 \mathrm{~K}$ & 0.085 & 0.085 & 0.108 & 0.152 & 0.187 \\
& $10 \mathrm{~K}$ & 0.343 & 0.435 & 0.572 & 1.54 & 1.6 \\
& $100 \mathrm{~K}$ & 2.75 & 4.61 & 6.63 & 15.0 & 27.1 \\
\hline \multirow{3}{*}{ PCADG } & $1 \mathrm{~K}$ & 0.315 & 0.759 & 1.46 & 3.17 & 9.05 \\
& $10 \mathrm{~K}$ & 2.09 & 10.07 & 14.47 & 32.1 & 77.2 \\
& $100 \mathrm{~K}$ & 22.10 & 64.50 & 166 & 207 & 1085 \\
\hline
\end{tabular}

Table 5.1: Running time (in seconds) of PADG and PCADG

\begin{tabular}{|l|l|l|l|l|l|l|}
\hline & Random & NRMP+ & SG & DG & PADG & PCADG \\
\hline Time (s) & 0.98 & 0.14 & 12.7 & 0.31 & 0.43 & 10.1 \\
\hline
\end{tabular}

Table 5.2: Running time (in seconds) of all algorithms with $10 \mathrm{~K}$ users and 100 events

Running Time. Finally, we evaluate the scalability of the algorithms by varying the number of users $(1 \mathrm{~K}, 10 \mathrm{~K}$ and $100 \mathrm{~K})$ and events $(50,100,150,200$, and 500). We maintain the default settings for all other parameters. Table 5.1 shows the running time of PADG and PCADG. Overall, both algorithms achieve good efficiency, finishing within seconds or minutes in all cases, and PADG always runs faster than PCADG. As the trend is similar, we show the running time comparison with Random, NRMP+, SG, and DG for one case of 10K users and 100 events. Random, DG, and SG takes $0.98,0.31$, and 12.7 seconds to finish, while PADG takes 0.43 and PCADG takes 10.07 seconds respectively (see Table 5.2). SG was consistently the slowest algorithm in all cases that we tested. For our largest simulation of $100 \mathrm{~K}$ users and 500 events, PCADG takes 20 mins. This is a reasonable for large scale event organization, where real-time response is not critical.
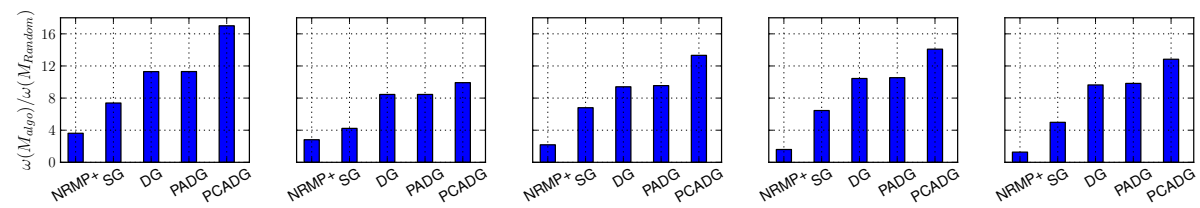

Figure 5.3: Improvement of social welfare yielded by various algorithms over Random 

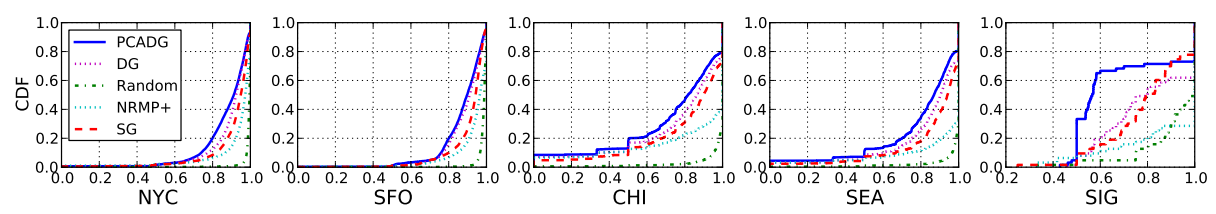

Figure 5.4: CDF showing regret ratio $\rho$ for different algorithms.

\subsection{Experiments on real event datasets}

\section{Data Description and Preprocessing}

We evaluate our algorithms on the following three real datasets. Plancast and Meetup are local event organization websites that allow groups of users to interact and plan events. We make use of the datasets ${ }^{1}$ released by Liu et al. [19]. Since in Plancast and Meetup users and events are from all over the world, we select several big cities and project the data to these specific locations. Our third dataset is SIGCOMM2009 (referred to as SIG), collected by Pietilainen et al. [22] and contains the bluetooth traces and social profiles (friends and interests) of 76 participants of a mobile application during the SIGCOMM conference at 2009. The following pre-processing was performed on the datasets to match our problem setting.

Plancast. We extract two subsets from the Plancast dataset in [19] corresponding to users and events in the vicinity of Chicago (CHI) and Seattle (SEA). The two datasets CHI and SEA have 2338 and 2327 users, and 339 and 360 events, respectively. For computing the innate affinity, we set $\sigma_{u, a}$ to 1 for events located within 0.01 units of Euclidian distance from the user's geolocation. Plancast allows users to subscribe to each other and receive updates on friends' activities, which gives us a ground-truth social network. We compute the Katz distance [12] from each user on the subscription graph to learn social affinity $w(u, v)$ 's. By definition of Katz, we have

$$
w(u, v):=\sum_{\ell=1}^{\infty} \alpha^{\ell} \cdot\left|P_{u, v}^{(\ell)}\right|
$$

where $P_{u, v}^{(\ell)}$ is the set of paths of length $\ell$ between $u$ and $v$ in the graph, and $\alpha$ is a

\footnotetext{
${ }^{1}$ http://www.largenetwork.org/ebsn
} 
damping factor so that the measure counts short path more heavily, which is set to 0.01 in our case. Higher Katz scores intuitively implies that two nodes are more connected, and hence gives a higher social affinity value for our purpose.

Meetup. Similarly, we project Meetup data from [19] on events and users located in San Francisco and New York City and refer to these datasets as SFO and NYC. SFO contains 6438 users and 59 events, while NYC contains 10328 users and 127 events. Meetup allows users to create and participate in groups, which in turn organize events. For both locations, we consider the labeled heterogeneous tripartite graph between users, groups, and events, with edges labeled by the relation that connects the pairs of nodes: e.g., if user $u$ is a member of group $g$ that organized event $a$ which the user attended, we draw edges $(u, g)$ and $(g, a)$. We then define $\sigma_{u, a}$ as the Katz distance between the user node $u$ and event node $a$ in the heterogeneous graph. where higher Katz score indicating higher affinity. For social affinity between users, we utilize tags provided in the data. More specifically, users on Meetup can attach tags in their profiles to indicate preferences and interests. Intuitively, users share common interests may enjoy being with each other in activities they both like. Thus, for each $(u, v) \in E$, we compute $w(u, v)$ as the Jaccard similarity coefficient of $u$ 's tag sets and $v$ 's tag sets:

$$
w(u, v):=\frac{\left|T_{u} \cap T_{v}\right|}{\left|T_{u} \cup T_{v}\right|}
$$

where $T_{u}$ and $T_{v}$ is are sets of tags of $u$ and $v$ respectively. Note that users without any tags in their profile have been filtered out.

SIGCOMM2009. This is a small dataset with 76 users, and 11 events, collected by a mobile application that records bluetooth device discovery information. Users' innate utility for events is 1 if a user attends an event as indicated by the data, and 0 otherwise. The data includes ground-truth Facebook friendship information, and thus as in the case of Plancast, we take this underlying social network and compute the Katz scores for social affinities.

Finally, for all five datasets described above, the user-event innate utility is set to 1 if the user attends the event indicated by the data, and to 0 otherwise. Also due to lack of ground-truth data, we generate the maximum and minimum cardi- 
nality constraints on the events as in the basic synthetic case, i.e., let $r$ be the ratio between the number of users and the number of events, for all $a \in A$, maximum cardinality constraint $\delta_{a}$ is sampled from a normal distribution with mean $2 r$ and variance $r$, while minimum cardinality $\gamma_{a}$ is sampled uniformly at random from $\left[1, \delta_{a}\right]$. Figure 5.2 shows the distributions of social affinity values for all datasets.

\section{Evaluations and Analysis}

Social Welfare. We evaluate the various algorithms on our objective, social welfare. Figure 5.3 shows the social welfare (Equation 3.2) obtained by the various methods considered relative to the welfare obtained using a Random assignment. Specifically, it shows the ratio between the social welfare yielded by assignments made by NRMP+, SG, DG, PADG, PCADG and that by Random. PCADG consistently has the highest social welfare, followed by PCADG, DG, SG and NRMP+. The margin between PCADG and the rest of the algorithms is significant in all cases, indicting its overall effectiveness. The biggest lead of PCADG is observed in NYC, 17 times better than Random, while the smallest happens in SIG, in which it is still 4 times better. On all datasets PADG and DG have similar welfare values, with PADG outperforming only slightly. This is because the generated minimum cardinality constraints for events are much smaller than maximum cardinality constraints. Recall from Figure 5.1 (a) that when $\gamma_{s}$ are high, the gap between PADG and DG is also high.

Regret Ratio. Figure 5.4 shows the cumulative distribution function (CDF) of the regret ratio $\rho(u)$ (see Equation 5.1) achieved by the various methods (PADG skipped for clarity) on the five datasets. The general trend is similar to that of social welfare, where PCADG outperforms all baselines. For instance, in NYC, $20 \%$ of the users have a regret below 0.8 using PCADG, while only $8 \%$ have that regret with NRMP+. This gap is even more pronounced in CHI and SEA, with the largest difference in SIG. In particular, $70 \%$ of users have a regret ratio below 0.7 using PCADG, while only $18 \%$ have that regret using NRMP+.

In summary, through extensive experiments on both synthetic and real world data, we have demonstrated the effectiveness (in terms of social welfare and regret) and efficiency (in terms of running time) of our proposed solution. 


\section{Chapter 6}

\section{Conclusions and future work}

While there has been considerable work on detecting emerging events from social media, the related, equally important area of organizing events has received much less research attention. This thesis provides a first systematic study towards the novel research direction of Social Event Organization (SEO), motivated by popular social event organization platforms like Meetup and Plancast and by applications such as organizing events using these platforms over a wide local area, and organizing events co-located with large conferences and conventions.

In this thesis, we understand the organziation as the assignment problem, where we consider two critical factors: innate affinity, which captures a user's intrinsic preferences for or interests in the events being offered, and social affinity, which captures the social connections among the users themselves. We formally define the overall measure of social welfare in event organization and formalize the SEO problem as an optimization problem for the social welfare with constraints on the participation cardinality of the events. We show that this problem is not only NPhard, but also hard to approximate. We propose a set of heuristic solutions that leverage the notion of phantom events and the technique of looking ahead the potenial gain that may accrue as a result of future assignments to the current event for which a user is being assigned. Using an extensive set of experiments over synthetic datasets and three real datasets including those from the platforms Meetup and Plancast, we demonstrate that our heuristic algorithms are scalable and furthermore outperform the baseline algorithms significantly in terms of achieving 
superior social welfare.

Furthermore, we would like to provide an landscape discussion on some interesting future directions to extend our problem formulation.

To start with, we could incorporate interdependency between innate and social affinities. E.g., Jack and Jill may be close friends but may not like to play poker together since they know each other's tells too well. Jill may like going to an ice hockey game in which she normally has low innate affinity, but with Mike who is knowledgeable and keeps her engaged with interesting anecdotes. Handling such interdependent affinities is important. Our framework could be extended by generalizing the innate affinity beyond what's between one individual person and one event, to two or three more person. For the above example, we would have a high innate affinity value between the event of hockey and the pair of Jill and Mike. Although strictly more difficult, our phantom community aware dynamic greedy strategy would still apply in this case, where the community effect is now conditioned on specific event.

Another natural extention is to extend the weight to the negative case. In terms of innate affinity, this refers to the case that a person is "forced" to go a a event that he doesn't want to attend. In terms of social affinity, this would mean the two person hate each other so much [7] that they don't want to appear in the same events. Mathematically, our phantom community aware dynamic greedy strategy would still work as usuall and moreover since these negative weights would seldomly pop up in the priority queue, they would naturally be avoided. However, some better heuristics can be designed towards the specific case. For example, we may need to pay special attention and keep track of these retricted assignments to prevent it from happenning during post processing, or alternatively we could follow the scheme in the Gale-Shapley matching [10] for NRMP and redistribute the negative assignment as an after step.

More complex social interaction could be considered in terms of computing the social affinity. E.g. the Ninja Turtles may want to fight the evil as a group of Four, missing any of the four would undermine the integrety of the group and decrease the general social welfare. To handle this, we would not only consider the affinity based on pair, but also on triplet, quadraplet, etc. Then we would carry on our dynamic greedy strategy and each time, compute the community effect based 
on different types of social affinity.

On the event side, there are also many factors we can incorporate to empower our model framework. Various dimensions of an event, e.g. where, when, what type of event can also be formally studied. We could consider the scenario where the events spans across a wide area in a metropolitan region, each can only happen at specific time of day/week, and each person has a set home or office location and certain busy schedule. Currently our framwork handles these preference in a "one fits all" scheme and understand all such preference as a numeric value. However to make the our solution better applies to these realistic situation, certain optimization strategy can be employed to accomodate it, e.g. we could preprocess the candidate assignment to filter out geoloigcally or temporally restricted assignments, or we could employ the divide and conqueuer scheme and compute assignment in geological parallelly.

In terms of adding the time dimension to our problem, we could also model "multiple shot" event participation where each person is allowed to attend more than one event. This is realistic when we consider performing event assignment on online event organization platform such as Meetup, where the system could suggest you an schedule of events over a whole month, or the entire year. A straighforward solution is to treat the unit assigned to an event as not a person, but a person at specific time window, which would generates an assignment that will "remove" the one shot assumption. Based upon this, we could adjust the length and starting point of the time window to make personalized prediction to based on one's frequency the schedule preference.

More complex interplay along the time dimension may also be considered, e.g. we could imagine the event assignment as a self adaptive dynamic process to model more general social or actitivity of human being. During the course of participating events, people may learn to change their innate and social affinity, to cooperate and form a group or break up from a group, and act strategically. we may eventually need to incorporate game theory to model the individual strategic behavior. For example, we could view the event participation as a repeated game [20] with specific cost or gains for each individual, and we could study how to make arrangements that is in accordance with nash equilibrium so that everyone is satiefied in both individual level while still taking into account the social welfare 
from a global point of view. Although this would extend far beyond our current model framework. 


\section{Bibliography}

[1] W. Aiello, F. Chung, and L. Lu. A random graph model for massive graphs. In Proceedings of the thirty-second annual ACM symposium on Theory of computing, pages 171-180. Acm, 2000. $\rightarrow$ pages 27

[2] S. Amer-Yahia, S. B. Roy, A. Chawla, G. Das, and C. Yu. Group recommendation: Semantics and efficiency. $P V L D B, 2(1), 2009$. $\rightarrow$ pages 8

[3] A. Anagnostopoulos, L. Becchetti, C. Castillo, A. Gionis, and S. Leonardi. Online team formation in social networks. In $W W W, 2012$. $\rightarrow$ pages 8

[4] N. Armenatzoglou, S. Papadopoulos, and D. Papadias. A general framework for geo-social query processing. Proceedings of the VLDB Endowment, 6 (10):913-924, 2013. $\rightarrow$ pages 9

[5] N. Armenatzoglou, H. Pham, V. Ntranos, D. Papadias, and C. Shahabi. Real-time multi-criteria social graph partitioning: A game theoretic approach. In Proceedings of the 2015 ACM SIGMOD International Conference on Management of Data, pages 1617-1628. ACM, 2015. $\rightarrow$ pages 9

[6] P. Biró, T. Fleiner, R. W. Irving, and D. Manlove. The college admissions problem with lower and common quotas. Theor. Comput. Sci, 411((34-36)): 3136-3153, 2010. $\rightarrow$ pages 6, 7

[7] M. J. Brzozowski, T. Hogg, and G. Szabo. Friends and foes: ideological social networking. In Proceedings of the SIGCHI conference on human factors in computing systems, pages 817-820. ACM, 2008. $\rightarrow$ pages 34

[8] C. Chekuri and S. Khanna. A ptas for the multiple knapsack problem. In SODA, 2000. $\rightarrow$ pages 4,6

[9] L. Fleischer, M. X. Goemans, V. S. Mirrokni, and M. Sviridenko. Tight approximation algorithms for maximum general assignment problems. In SODA, 2006. $\rightarrow$ pages 4,6 
[10] D. Gale and L. S. Shapley. College admissions and the stability of marriage. Amer. Math. Monthly, 69, 1962. $\rightarrow$ pages 6, 18, 34

[11] K. Hamada, K. Iwama, and S.Miyazaki. The hospitals/ residents problem with quota lower bounds. In MATCH-UP: Matching Under Preferences Workshop at ICALP, 2008. $\rightarrow$ pages 6,7

[12] L. Katz. A new status index derived from sociometric analysis. Psychometrika, 18(1), 1953. ISSN 0033-3123. $\rightarrow$ pages 30

[13] S. Khot. On the power of unique 2-prover 1-round games. STOC, 2002. $\rightarrow$ pages 15

[14] J. Kleinberg and E. Tardos. Approximation algorithms for classification problems with pairwise relationships: Metric labeling and markov random fields. Journal of the ACM (JACM), 49(5):616-639, 2002. $\rightarrow$ pages 9

[15] S. O. Krumke and C. Thielen. The generalized assignment problem with minimum quantities. European Journal of Operational Research, 2013. $\rightarrow$ pages $4,6,13,14$

[16] T. Lappas, K. Liu, and E. Terzi. Finding a team of experts in social networks. In $K D D, 2009 . \rightarrow$ pages 8

[17] K. Li, W. Lu, S. Bhagat, L. V. Lakshmanan, and C. Yu. On social event organization. In Proceedings of the 20th ACM SIGKDD international conference on Knowledge discovery and data mining, pages 1206-1215. ACM, 2014. $\rightarrow$ pages iii

[18] D. Liben-Nowell and J. Kleinberg. The link-prediction problem for social networks. Journal of the American society for information science and technology, 58(7):1019-1031, 2007. $\rightarrow$ pages 11

[19] X. Liu, Q. He, Y. Tian, W.-C. Lee, J. McPherson, and J. Han. Event-based social networks: linking the online and offline social worlds. In KDD, 2012. $\rightarrow$ pages 30,31

[20] J.-F. Mertens. Repeated games. Springer, 1989. $\rightarrow$ pages 35

[21] E. Petrank. The hardness of approximations : Gap location. Computational Complexity, 4:133-157, 1994. $\rightarrow$ pages 14

[22] A.-K. Pietilainen and C. Diot. CRAWDAD data set thlab/sigcomm2009 (v. 2012-07-15). Downloaded from http://crawdad.cs.dartmouth.edu/thlab/sigcomm2009, 2012. $\rightarrow$ pages 30 
[23] P. Raghavendra and D. Steurer. Graph expansion and the unique games conjecture. In STOC, 2010. $\rightarrow$ pages 15

[24] A. E. Roth. The evolution of the labor market for medical interns and residents: a case study in game theory. Journal of Political Economy, 6(4): 991-1016, 1984. $\rightarrow$ pages 4, 6

[25] D. B. Shmoys and É. Tardos. An approximation algorithm for the generalized assignment problem. Math. Program., 62:461-474, 1993. $\rightarrow$ pages 4,6

[26] M. Sozio and A. Gionis. The community-search problem and how to plan a successful cocktail party. In Proceedings of the 16th ACM SIGKDD international conference on Knowledge discovery and data mining, pages 939-948. ACM, 2010. $\rightarrow$ pages 4, 9 